$12-29-2020$

\title{
Red Panda: A Novel Method for Detecting Variants in Single-Cell RNA Sequencing
}

\author{
Adam Cornish \\ University of Nebraska Medical Center \\ Shrabasti Roychoudhury \\ University of Nebraska Medical Center \\ Krishna Sarma \\ University of Nebraska Medical Center \\ Suravi Pramanik \\ University of Nebraska Medical Center, suravi.pramanik@unmc.edu \\ Kishor Bhakat \\ University of Nebraska Medical Center, kishor.bhakat@unmc.edu
}

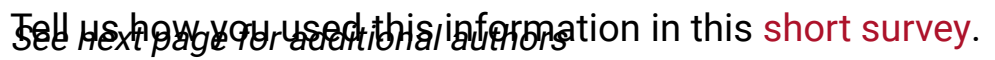

Follow this and additional works at: https://digitalcommons.unmc.edu/com_gcba_articles

Part of the Medical Anatomy Commons, Medical Cell Biology Commons, and the Medical Genetics Commons

\section{Recommended Citation}

Cornish, Adam; Roychoudhury, Shrabasti; Sarma, Krishna; Pramanik, Suravi; Bhakat, Kishor; Dudley, A T.; Mishra, Nitish K.; and Guda, Chittibabu, "Red Panda: A Novel Method for Detecting Variants in Single-Cell RNA Sequencing" (2020). Journal Articles: Genetics, Cell Biology \& Anatomy. 46.

https://digitalcommons.unmc.edu/com_gcba_articles/46

This Article is brought to you for free and open access by the Genetics, Cell Biology \& Anatomy at DigitalCommons@UNMC. It has been accepted for inclusion in Journal Articles: Genetics, Cell Biology \& Anatomy by an authorized administrator of DigitalCommons@UNMC. For more information, please contact digitalcommons@unmc.edu. 


\section{Authors}

Adam Cornish, Shrabasti Roychoudhury, Krishna Sarma, Suravi Pramanik, Kishor Bhakat, A T. Dudley, Nitish K. Mishra, and Chittibabu Guda 


\title{
Red panda: a novel method for detecting variants in single-cell RNA sequencing
}

\author{
Adam Cornish, Shrabasti Roychoudhury, Krishna Sarma, Suravi Pramanik, Kishor Bhakat, Andrew Dudley, \\ Nitish K. Mishra and Chittibabu Guda (i)
}

From The International Conference on Intelligent Biology and Medicine (ICIBM) 2020

Virtual. 9-10 August 2020

\begin{abstract}
Background: Single-cell sequencing enables us to better understand genetic diseases, such as cancer or autoimmune disorders, which are often affected by changes in rare cells. Currently, no existing software is aimed at identifying single nucleotide variations or micro (1-50 bp) insertions and deletions in single-cell RNA sequencing (scRNA-seq) data. Generating high-quality variant data is vital to the study of the aforementioned diseases, among others.

Results: In this study, we report the design and implementation of Red Panda, a novel method to accurately identify variants in scRNA-seq data. Variants were called on scRNA-seq data from human articular chondrocytes, mouse embryonic fibroblasts (MEFs), and simulated data stemming from the MEF alignments. Red Panda had the highest Positive Predictive Value at 45.0\%, while other tools_FreeBayes, GATK HaplotypeCaller, GATK UnifiedGenotyper, Monovar, and Platypus_ranged from 5.8-41.53\%. From the simulated data, Red Panda had the highest sensitivity at $72.44 \%$.
\end{abstract}

Conclusions: We show that our method provides a novel and improved mechanism to identify variants in scRNAseq as compared to currently existing software. However, methods for identification of genomic variants using scRNA-seq data can be still improved.

Keywords: Red panda, Variant calling using scRNAseq, Single cell sequencing, Human articular chondrocytes, Heterozygous variant calling

\section{Background}

Single-cell sequencing (SCS) is a relatively new technique that saw its first use in 2011 [1] and has been used to investigate important biological problems: examining the heterogeneity of different cancers [2], determining copy number variation in enhanced detail [3], and better characterizing circulating tumor cells using differential expression analysis [4, 5]. Multiple recent studies using SCS have also shown that tumors are genetically diverse

\footnotetext{
* Correspondence: babu.guda@unmc.edu

Department of Genetics, Cell Biology and Anatomy, University of Nebraska Medical Center, Omaha, NE 68198, USA
}

and produce subclones that contribute to the pathogenicity of the disease by conferring chemotherapy resistance and metastatic capabilities to the tumor [6, 7]. This technology has also proven useful by aiding in characterizing somatic mutations in neurons [8], identifying rare intestinal cell types [9], and discriminating cell types in healthy tissues $[10,11]$.

One area that has not been widely explored is the detection of small variants in SCS. Single Nucleotide Variants (SNVs) and micro (1-50 bp) insertions and deletions (indels) can have a large impact on human disease [12-14] and are typically identified using exome

(c) The Author(s). 2020 Open Access This article is licensed under a Creative Commons Attribution 4.0 International License which permits use, sharing, adaptation, distribution and reproduction in any medium or format, as long as you give appropriate credit to the original author(s) and the source, provide a link to the Creative Commons licence, and indicate if changes were made. The images or other third party material in this article are included in the article's Creative Commons licence, unless indicated otherwise in a credit line to the material. If material is not included in the article's Creative Commons licence and your intended use is not permitted by statutory regulation or exceeds the permitted use, you will need to obtain permission directly from the copyright holder. To view a copy of this licence, visit http://creativecommons.org/licenses/by/4.0/. The Creative Commons Public Domain Dedication waiver (http://creativecommons.org/publicdomain/zero/1.0/) applies to the data made available in this article, unless otherwise stated in a credit line to the data. 
sequencing or whole-genome sequencing (WGS) [15]. There are few available tools for SNVs identification, which will work for bulk RNA-seq data [16, 17]. Monovar has been developed to identified variants in scDNAseq [18], but there exists no companion tool for scRNAseq. An effort has been made to apply best practices for identifying variants in RNA-seq to scRNA-seq datasets $[8,19]$, but they do not take advantage of the unique nature of the data produced by the scRNA-seq platform.

This study introduces a novel method, Red Panda, that is designed specifically to identify variants in single-cell RNA sequencing (scRNA-seq) and tests how it compares to currently-available variant callers: FreeBayes [20], GATK HaplotypeCaller [21], GATK UnifiedGenotyper [22], Platypus [23], and Monovar [18]. The first four tools were originally developed for calling variants using bulk DNA sequencing data but can also identify variants in bulk mRNA sequencing data. For our purposes, data from scRNA-seq, as opposed to scDNA-seq, is used as it largely avoids errors stemming from single-cell genomic sequencing-high allelic dropout, coverage nonuniformity leading to lack of coverage in exons, and False Positive (FP) amplification errors [24].

Red Panda employs the unique information found in scRNA-seq to increase accuracy as compared to software designed for bulk sequencing. We utilize the fact that transcripts represented by scRNA-seq reads necessarily only originate from the chromosomes present in a single cell. Where applicable, this fact is used to decide what is and is not a heterozygous variant. For example, if $20 \%$ of the transcripts of a gene originate from the maternal chromosome and $80 \%$ originate from the paternal, then all the heterozygous variants of that gene in the expressed transcript will be represented at a reference to alternate allele ratio of either 1: 4 or 4:1. In other words, all the heterozygous variants in that transcript are expected to be part of a bimodal distribution, which can be exploited to improve the accuracy of variant calling using scRNA-seq data. Such unique information could not be obtained from bulk sequencing, where each variant is independently called. As part of the process of identifying variants, Red Panda creates three different classes: homozygous-looking, bimodally-distributed heterozygous, and non-bimodally-distributed heterozygous. We use simulated and experimental data to prove that this partitioning strategy, as well as treating bimodally-distributed variants differently, leads to an increase in sensitivity and Positive Predictive Value (PPV) compared to currently available methods. A preprint of Red Panda is available at https:// www.biorxiv.org/content/10.1101/2020.01.08.898874v2 [25].

\section{Methods}

\section{Data generation and quality control}

For algorithm development, human articular chondrocytes were sequenced using the Smart-seq2 protocol for single cells (Supplemental Fig. 1). These data satisfied five criteria needed for a test dataset: (i) bulk genomic sequencing data paired with scRNA-seq data generated from Smart-seq2 libraries, (ii) isogenic tissue, (iii) high quality sequencing data, (iv) the sequencing data must be from an organism with a well-annotated genome, (v) the sequencing data must come from normal cells. The first criterion was especially important because the bulk sequencing data was used to corroborate the findings from the scRNA-seq data. For this dataset, 30 live cells successfully captured from a 96 chamber $\mathrm{C} 1$ Fluidigm IFC were sequenced and eight were removed due to: low read count, too many reads originating outside exons (percentage of reads outside exons is one standard deviation above the median percentage of reads outside exons for all samples), and/or transcription profiles not correlating with the other cells sequenced (Pearson's Correlation: $p>0.05$; Supplemental Figs. $2 \& 3$ and Supplemental Table 1).

Additionally, 56 mouse embryonic fibroblasts (MEFs) were sequenced using the Smart-seq2 protocol and are paired with Sanger sequencing for validation. Simulated data were generated from the MEF alignment files for each sample (Supplemental Fig. 4). Of these, one cell was removed due to its low read counts, too many reads originating outside exons, and transcription profiles not correlating with the other cells sequenced (Supplemental Figs. 5 \& 6).

\section{Exome sequencing}

We performed cell prep, and DNA extraction on human articular chondrocytes harvested the same day from the same batch as the single-cell capture. Genomic DNA was extracted using the QIAGEN DNA extraction kit per manufacturer's instructions. Due to the low amount of DNA captured (80 ng), 12 PCR amplification cycles were performed prior to library preparation to obtain enough DNA. The Agilent SureSelect Clinical Research Exome V2 kit was used to capture coding regions and generate a library. The exome library was sequenced on two lanes of the NextSeq500 using 75 base pair paired-end sequencing. Whole exome sequencing statistics of the articular chondrocyte are provided in Supplemental Table 2.

\section{Exome variant calling}

The bcbio-nextgen v. 1.0.3 pipeline was used for variant calling to align reads and identify variants in the exome. Reads were aligned to the human genome v. 38 (hg38) using BWA-MEM v. 0.7.15. FreeBayes (v. 1.1.0), GATK HaplotypeCaller (v. 3.7.0), and Platypus (v. 0.8.1) were used to identify SNVs and indels. Only those variants identified by at least two out of the three algorithms were kept (Supplemental Table 3). MultiQC v. 1.0.dev0 was run to aggregate Quality Control (QC) statistics from bcbio-nextgen, samtools v. 1.4, bcftools v. 1.4, and FastQC v. 0.11.5. 


\section{Single-cell RNA sequencing}

\section{Human articular chondrocytes}

Articular chondrocytes were harvested from a Caucasian female patient undergoing total knee replacement, who provided informed consent under IRB \#691-13-EP prior to the study. Cells were extracted from shavings of articular cartilage, all of which was consumed in the generation of the scRNA-seq and exome libraries. This was done through sequential digestion in $.2 \%$ Pronase (Roche) for $2 \mathrm{~h}$, followed by overnight digestion in .2\% collagenase (Gibco), all while shaking at $37^{\circ} \mathrm{C}$. Cell suspensions were passed through $70 \mu \mathrm{M}$ cell strainers (BD Falcon) and centrifuged at $500 \mathrm{xG}$ for $10 \mathrm{~min}$ to recover chondrocytes. The cells were subsequently embedded in threedimensional alginate bead cultures at a final concentration of about 75 million cells per mL. The cultures were maintained at $37^{\circ} \mathrm{C}$ in a $5 \% \mathrm{CO} 2$ atmosphere in Dulbecco's modified Eagle medium (DMEM)/F12 (1:1) supplemented with $1 \%$ penicillin-streptomycinglutamine (Invitrogen, 10,378-016), Amphotericin B (Gibco, 15,290,026), insulin-transferrin-sodium selenite (Sigma, I2771), $50 \mu \mathrm{g} / \mathrm{mL}$ Vitamin C, $10 \mathrm{ng} / \mathrm{mL}$ FGF2, and $10 \mathrm{ng} / \mathrm{mL}$ TGF-bb3 (PeproTech ${ }^{\circ}, 100-36 \mathrm{E}$ ) for 14 days. The day before single-cell capture, cells were lysed using Trizol $^{\odot}$ reagent (Life Technologies) according to the manufacturer's protocol. These cells were split into two groups for DNA and RNA extraction. Cells were loaded onto a $10-17 \mu \mathrm{m}$ Fluidigm C1 Single-Cell Auto Prep IFC, and the cell-loading script was performed using the manufacturer's instructions. Each of the 96 capture sites was inspected under a confocal microscope to remove sites containing dead cells (as identified by the LIVE/DEAD Cell Viability Assay) and to remove capture sites containing more than one cell. Cells that were not identified as either alive or dead by the LIVE/DEAD assay were retained for RNA sequencing. Summary of the human articular chondrocytes captured on the Fluidigm $\mathrm{C} 1$ is available in Supplemental Table 4. Following capture, reverse transcription and cDNA amplification were performed in the $\mathrm{C} 1$ system using the Clontech SMARTer Ultra Low Input RNA Kit for Sequencing v3 per the manufacturer's instructions. Amplification was performed using the Nextera XT DNA Sample Preparation Kit, and the Nextera XT DNA Sample Preparation Index Kit (Illumina) was used for indexing. After quantification using an Agilent Bioanalyzer, sequencing was performed on two lanes of the NextSeq500 using 150 base pair paired-end sequencing.

\section{Mouse embryonic fibroblasts}

Mouse embryonic fibroblasts (MEFs) were harvested from embryos at E13.5 and extracted using previously standardized methods [26]. After isolation, cells were cultured in DMEM containing $10 \%$ FBS and $1 \%$ of each penicillin and streptomycin at $37{ }^{\circ} \mathrm{C}$ in a $5 \% \mathrm{CO} 2$ atmosphere for 2 days. On the day of single-cell capture, cells were trypsinized (0.05\% Trypsin-EDTA solution), counted, and resuspended in media at 105 cells/mL concentration. Sequencing was performed as described above.

\section{Single-cell RNA variant calling}

The bcbio-nextgen v. 1.0.3 pipeline for RNA-seq was used to align reads and perform transcript quantification for each cell. Reads were aligned using hisat2 v. 2.1.0 to be used in the downstream analysis for FreeBayes, GATK HaplotypeCaller, GATK UnifiedGenotyper, Monovar, and Red Panda. However, for Platypus, BWAMEM v. 0.7.15 was used to align reads due to Platypus's inability to process reads split across long distances. The genome hg38 was used for the human articular chondrocytes, and mm10 was used for the MEFs. All four bulk variant callers were run using default parameters with a few exceptions. For FreeBayes, min-alternate-fraction was set to 0.1 and no-partial-observations was enabled, and GATK HaplotypeCaller and GATK UnifiedGenotyper set standard minimum confidence threshold for calling to 4.0. Sailfish v. 0.10 .1 was used to generate expression values. MultiQC v. 1.0.dev0 was run to aggregate QC statistics from bcbio-nextgen, samtools v. 1.4, QualiMap v. 2.2.2a53, and FastQCv. 0.11.5.

\section{Sanger sequencing}

Primer3Plus (Supplemental Table 5) was used to create target regions for Sanger sequencing. For the first round of sequencing, the PCR reaction was performed using GoTaq Hot Start Polymerase following the manufacturer's protocol with an annealing temperature (Tm) of $50{ }^{\circ} \mathrm{C}$. After amplification, PCR products were run on $1.5 \%$ agarose gel and visualized in Kodak gel doc, and specific DNA bands were recovered using QIAquick Gel Extraction Kit. For the second round of sequencing of the Red Panda-specific variants, a $55^{\circ} \mathrm{C}$ Tm was used, followed by running the PCR products on $2 \%$ agarose gel. Purified DNA products paired were submitted to Genewiz for Sanger sequencing.

\section{Statistic calculations}

For our calculations, (i) a True Positive (TP) is a position on the genome that is correctly identified as differing from the reference genome, (ii) a True Negative (TN) is a position on the genome that is correctly identified as not differing from the reference, (iii) a False Positive (FP) is a position on the genome that is incorrectly identified as differing from the reference, (iv) a False Negative $(\mathrm{FN})$ is a position on the genome that is incorrectly identified as not differing from the reference genome. 


$$
\text { Sensitivity }=T P /(T P+F N)
$$

\section{Positive Predictive Value $(P P V)=T P /(T P+F P)$}

\section{Software requirements and distribution}

Red Panda, written almost entirely in Perl, relies on samtools mpileup and GATK HaplotypeCaller to function. The tool mpileup is required to generate a list of every variant in a sample. GATK HaplotypeCaller is necessary to call heterozygous variants that do not fit a bimodal distribution. Bedtools, vcf-sort found in the vcftools package, and Picard Tools are all necessary to manipulate the different types of files used during the variant calling process. As these tools are all supported by different institutions under different licenses, Red Panda does not come prepackaged with them.

\section{Red panda algorithm}

Red Panda takes two files as input: a tab-delimited file generated by sailfish [27] containing a list of all isoforms and their expression levels in a cell and also a Variant Call Format (VCF) file generated by samtools mpileup [28] containing a pileup of all locations in the cell's genome that differ from the reference. The second file is the list of all putative variants from which Red Panda will create three lists: homozygous-looking, bimodallydistributed heterozygous, and non-bimodally-distributed heterozygous variants.

The distinction between heterozygous variants and homozygous-looking variants is necessary, because variants will either have a fraction of reads that support an unambiguously heterozygous variant, or they will have a fraction of reads that, in a single cell, appears to be a homozygous variant, but could potentially be heterozygous. This is due to the stochastic nature of RNA transcription leading to allele-specific expression [29-31]. This monoallelic expression can lead to a heterozygous variant looking like a homozygous variant [32, 33]. Due to this ambiguity, variants that have full read coverage supporting an alternate allele are hereafter termed "homozygous-looking" rather than "homozygous". The workflow for this methodology can be found in Fig. 1.

Red Panda capitalizes on the fact that read data come from a single cell, so transcripts represented by the scRNA-seq reads necessarily come from the two chromosomes present. It is this information that we factor that into our decision-making process when establishing what is and is not a variant. In a diploid cell, one would expect transcripts to originate from two chromosomes, and thus, any heterozygous variant present in a given transcript will be represented in the sequencing data by a fraction consistent with the fraction of transcripts coming from a specific chromosome. Figure 2 shows that if $30 \%$ of the transcripts for a gene in a cell originate from the maternal chromosome and 70\% from the paternal chromosome, then reads in the scRNA-seq data will represent every heterozygous variant present in that transcript at either a 7:3 ratio (reference: alternate allele) or a 3:7 ratio. This type of variant is considered to be bimodally-distributed heterozygous and any variant on the same transcript that's falling outside of this distribution with a tolerance of $5 \%$ is likely to be a False Positive. Using this concept, Red Panda can accurately remove False Positive heterozygous variant calls-often artifacts from the library preparation, sequencing, or alignmentas well as identify variants supported by even a low fraction of reads that the current tools would not be able to capture (Supplemental Fig. 7).

The above described bimodally-distributed heterozygous variants are processed from the VCF file generated by samtools mpileup. This file is split into two lists containing variants that are heterozygous or homozygouslooking. Heterozygous variants are filtered into two files: one containing the aforementioned bimodally-distributed variants and one containing the non-bimodallydistributed variants, the latter of which is filtered by GATK HaplotypeCaller as there is no unique information that Red Panda can capitalize on. GATK HaplotypeCaller is used because it has been proven to be among the most accurate variant callers available [34].

The final list of variants that is presented to the user contains those that: are heterozygous and fit a bimodal distribution, are heterozygous and did not fit a bimodal distribution but were supported by GATK HaplotypeCaller, and those that appeared to be homozygous and had a read depth of at least 10x. This method of partitioning variants is also used for indels.

Red Panda runs on a single core but can easily be parallelized by being run on a cluster. Each cell, each with $\sim 5.4$ million reads, takes, on average, two hours to complete the analysis.

\section{Simulation}

Roughly 1000 simulated variants were programmatically inserted into the alignments generated from the MEFs resulting in a unique set of simulated variants for each cell. Of these, 650 were homozygous, and $\sim 350$ were heterozygous, a subset of which $\sim 70$ were bimodallydistributed. These numbers were used because they are close to the proportions seen in the variants corroborated by the exome sequencing in the articular chondrocyte data. While these proportions do not match those expected based on bulk sequencing experiments [35-37], they do match what is expected from scRNA-seq data [19]. 


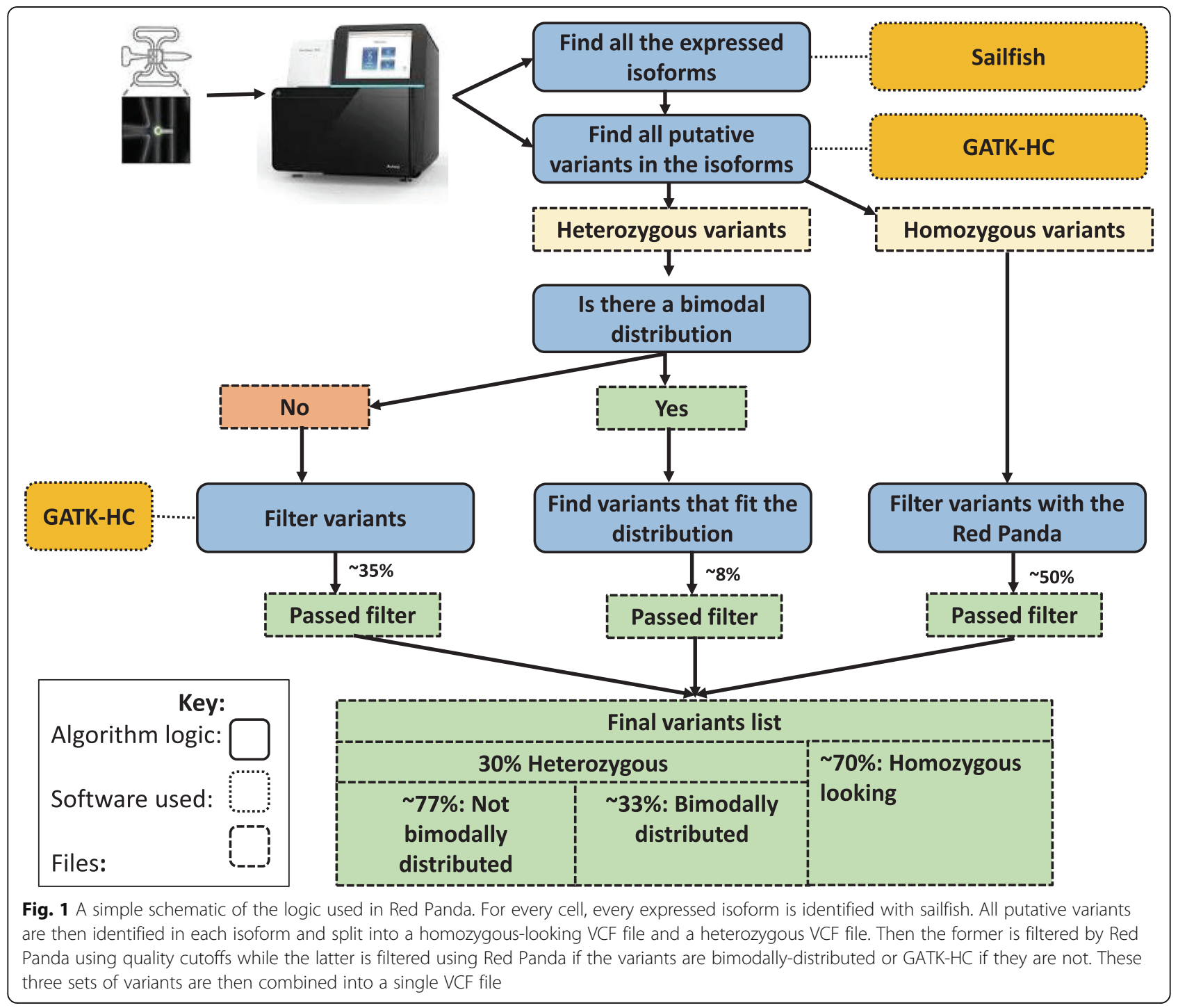

To instill a level of uniformity, a list of locations was randomly selected from the alignment files where there was a read depth of at least 20x (Supplemental Fig. 8). The positions on the genome where the 650 homozygous and 280 non-bimodally-distributed heterozygous variants were inserted were not restricted except that they must originate from the locations with at least 20x read coverage. Conversely, the bimodally-distributed heterozygous variants had additional parameters determining their placement. They were required to have a minimum of two variants placed in an expressed (TPM $>1$ ) isoform. From the MEF sequence data, an average of $\sim 3$ (a range of 25) variants per isoform were observed resulting in 23 randomly chosen genes being used for this class of variant. For each isoform, $2-5$ variants were randomly inserted into the gene but only if more than $250 \mathrm{bp}$ of viable (read depth $>=20 x$ ) locations existed.

\section{Results}

Comparison of different tools using human articular chondrocytes

Alignment files generated for each of the 22 chondrocyte scRNA-seq samples were used as input for FreeBayes, GATK HaplotypeCaller, GATK UnifiedGenotyper, Monovar, Platypus, and Red Panda. The variant calls generated by each tool were cross-referenced with the variants found in the exome to determine their veracity. To avoid False Negatives, comparisons were restricted to locations supported by alignments in both the exome and the cell being compared. To evaluate the ability of each method, the number of variants found in concordance with the exome as well as the PPV for each tool were calculated.

Figure 3a shows that, on average, Red Panda identifies 913 variants per cell that are in accordance with the exome whereas FreeBayes identifies 65, GATK HaplotypeCaller 705, GATK UnifiedGenotyper 222, Monovar 861, 


\section{Finding a bimodal distribution}

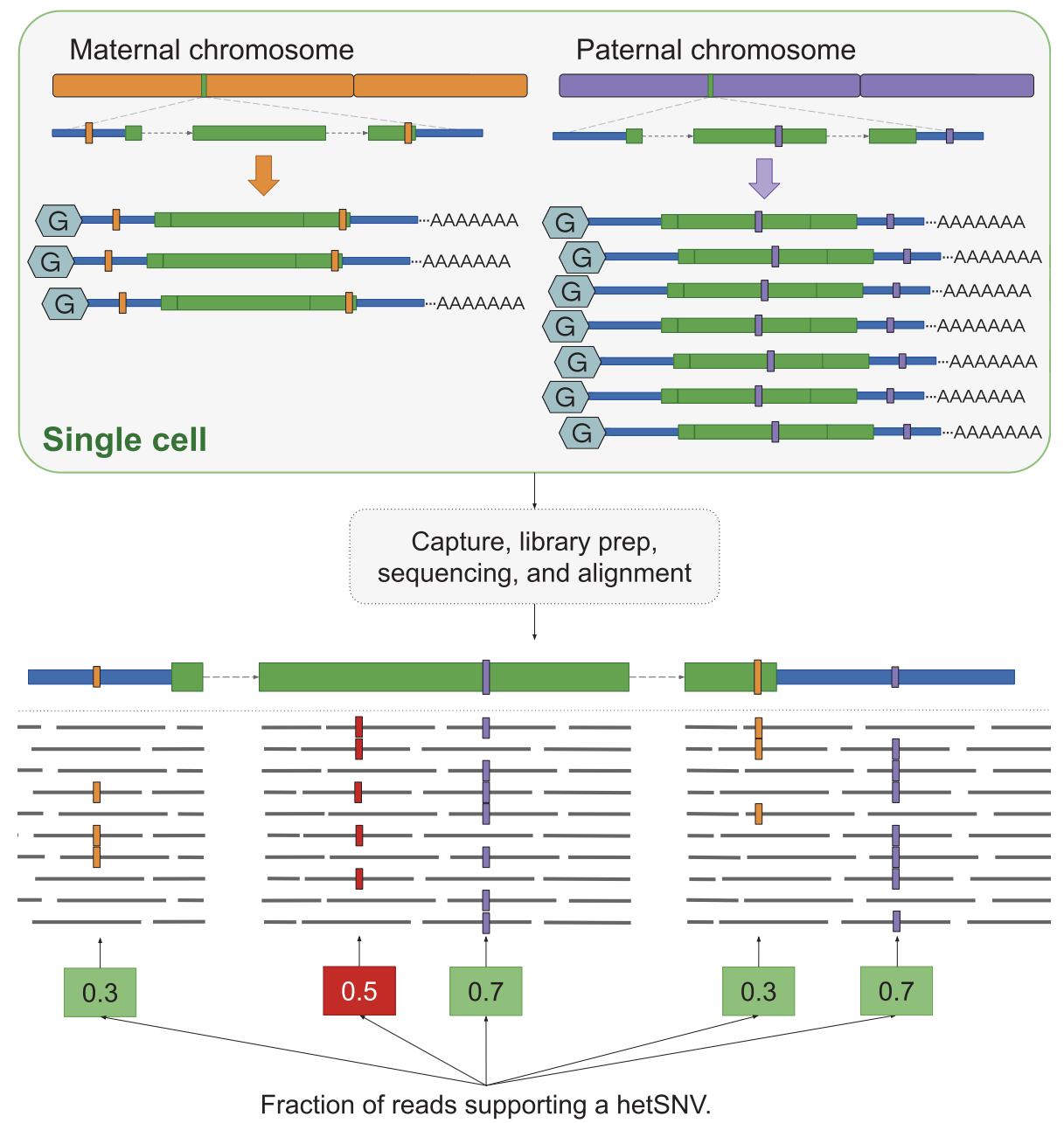

Fig. 2 Finding a bimodal distribution. Any variants (green box) that fit into the expected distribution of reads stay. Any that do not are removed: here the variant existing at a fraction of 0.5 (red box) would be removed

and Platypus 386. There is a consistent overlap between the tools, even for FreeBayes and GATK UnifiedGenotyper which typically did not identify as many variants as the other tools (Supplemental Fig. 9). While Red Panda shares significant overlap with the other tools, it also identifies a large number of unique variants by itself.

To assess the effectiveness of these variant callers with regards to heterozygous variant identification, the same analysis was performed using just the heterozygous SNVs and indels in each sample. Each tool has its own annotation dictating if a variant is heterozygous, and these variants were cross-referenced with the exome sequencing data to confirm the variants were, in fact, heterozygous.

Figure $3 \mathrm{~b}$ shows the total number of heterozygous SNVs and indels in concordance with the exome for each tool and each cell. Percent of variants that are heterozygous and validated by GATK-HC, and heterozygous and validated by Red Panda are provided in Supplemental Table 6 . On average, 154 variants in agreement with the exome were identified by Red Panda, 31 by FreeBayes, 136 by GATK HaplotypeCaller, 118 by GATK UnifiedGenotyper, 368 by Monovar, and 36 by Platypus. PPV and False Discovery Rate (FDR) were calculated (Table 1 and Fig. 3c), and show that Red Panda has the highest average PPV (44.96\%) of any of the tools.

\section{Comparison of different tools using MEFs}

The six software packages were compared by assessing the variant overlap between cells. As these cells were isogenic, each cell should have shared a large portion of its variants with the other cells sequenced. This was evaluated with a high overlap identified by a 


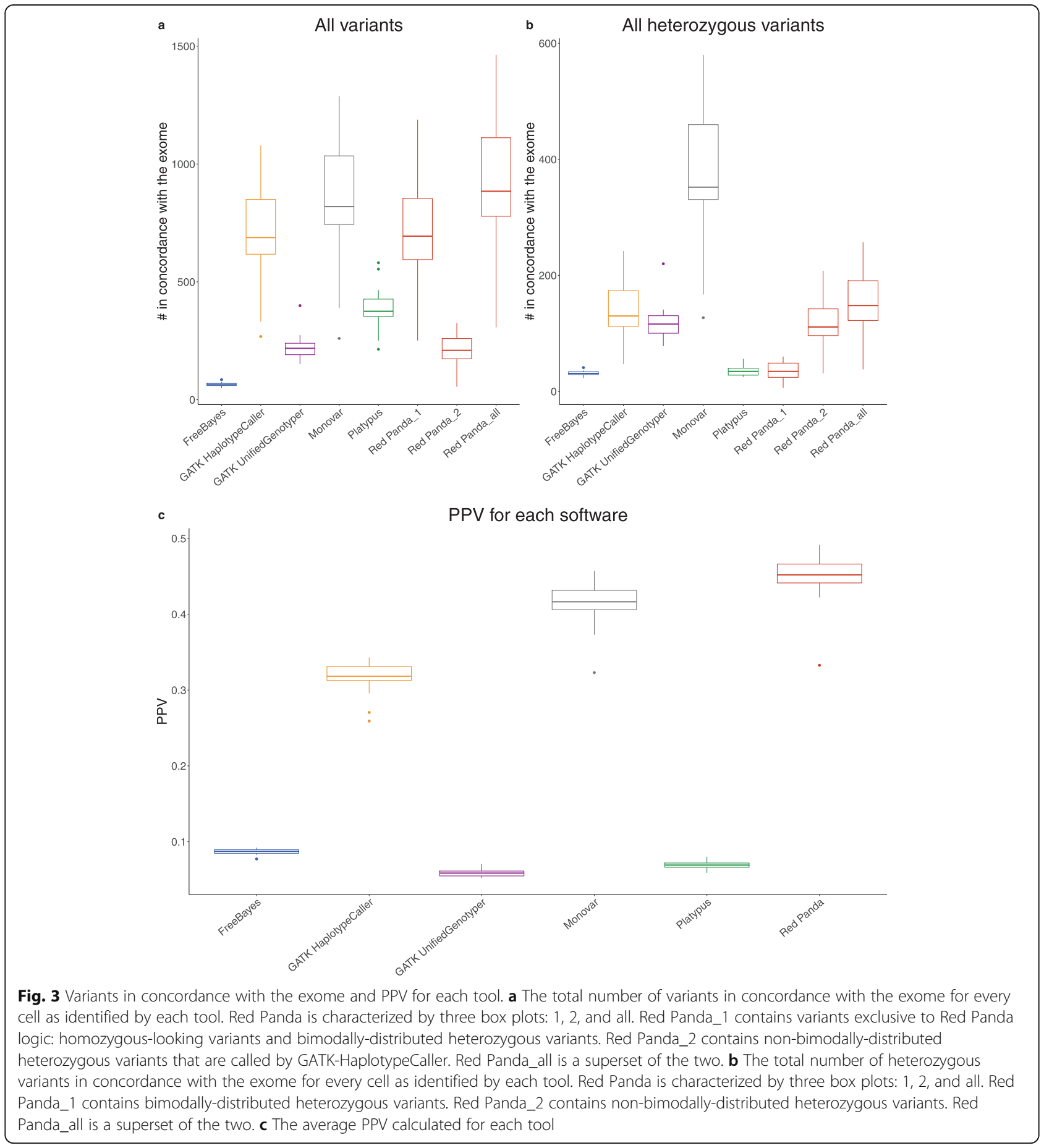

variant caller as an indicator that that software performed well.

Table 2 shows the average number of variants identified with Monovar identifying the highest number of variants of all the tools. Extrapolating from the PPV results from the articular chondrocyte data, this means that Monovar also identified the highest number of True Positives and fewest False Positives.
Overlap of variants between cells was measured by looking at the all-to-all comparison of 55 cells, resulting in 1540 unique possible comparisons. Three groups of variants are assessed in these comparisons: all variants shared, homozygous-looking variants shared, and heterozygous variants shared (Supplemental Figs. 10 \& 11).

Figure 4 shows the distribution of the fraction and a total number of variants overlapping in the pairwise 
Table 1 PPV and FDR for each tool

\begin{tabular}{lll}
\hline Algorithm & Average PPV (\%) & Average FDR (\%) \\
\hline FreeBayes & $8.69 \% \pm 0.35 \%$ & $91.31 \% \pm 0.35 \%$ \\
GATK HaplotypeCaller & $31.67 \% \pm 2.08 \%$ & $68.33 \% \pm 2.08 \%$ \\
GATK UnifiedGenotyper & $5.84 \% \pm 0.45 \%$ & $94.16 \% \pm 0.45 \%$ \\
Monovar & $41.53 \% \pm 0.29 \%$ & $58.47 \% \pm 0.29 \%$ \\
Platypus & $6.95 \% \pm 0.49 \%$ & $93.05 \% \pm 0.49 \%$ \\
Red Panda & $44.96 \% \pm 3.15 \%$ & $55.04 \% \pm 3.15 \%$ \\
\hline
\end{tabular}

The average PPV and FDR with standard deviations for each tool using the exome as a reference is listed

comparisons for all three classes of variants. Monovar identifies both the highest fraction of variants shared in pairwise comparisons, as well as total variants shared. For Red Panda, the highest fraction and total count of variants shared in pairwise comparisons come from the homozygous-looking class wherein more than $75 \%$ of the comparisons achieve a higher fraction of overlap than every other tool. Interestingly, it is rare for any tool to have more than 100 heterozygous variants shared between cells.

\section{Validation with simulated data}

Sensitivity was calculated for each tool across every cell. Figure 5 and Supplemental Fig. 12 show that for homozygous variants and bimodally-distributed heterozygous variants, Red Panda consistently outperforms the other four tools, resulting in a higher overall sensitivity. For heterozygous variants taken as a whole, Monovar performs the best of the tools. It is unsurprising then that, compared to Monovar, Red Panda does not perform as well in this category because it uses GATK HaplotypeCaller (shown to accurately only a identify few heterozygous variants in this simulation) to validate heterozygous variants that do not follow a bimodal distribution. In this instance, GATK HaplotypeCaller and GATK UnifiedGenotyper perform poorly because they both utilize a feature that considers all samples simultaneously. This results in inferior performance on a group of samples where each sample may have a large number of mutations unique to that sample, and for this simulation, every cell has a 1000 variants unique to it. Red Panda does not suffer as much from this limitation as it explicitly directs GATK-HC to call variants at specific locations one at a time rather than jointly. However, this can result in lowered sensitivity for Red Panda as compared to Monovar on samples that are genetically similar where the latter identifies the highest fraction (Fig. 4e) and highest total number (Fig. 4f) of heterozygous variants shared in pairwise comparisons.

\section{Validation with sanger sequencing}

Sanger validation was performed on two sets of random variants found in the MEF sequencing: one set of 20 random variants identified by all variant callers, and one set of 20 random variants identified exclusively by Red Panda. The first group is meant to assess the accuracy of all the tools taken as a whole, and the second is to address whether the Red Panda-specific variants are reliable. One requirement of the variants being validated is that they were identified in at least two cells. Ideally, variants present in more than $50 \%$ of the cells would be chosen, but as Table 3 shows, there were not enough variants that are present in even $>22$ out of 55 of the cells to perform validation in this way.

Enough valid sequences were generated for 33 of the 40 targets by Sanger sequencing to validate the presence of their corresponding variant (Supplemental Tables 7, 8, 9). Of these 33, only three variants, all of which were exclusively identified by Red Panda, were validated by Sanger sequencing. In all three instances, the variants were found in nine or more cells.

\section{Discussion}

Identification of SNVs and indels is vital in addressing biological problems with a genetic component. While variant calling methods exist for samples collected from bulk sequencing, it is also important to have methods designed for samples collected from SCS. As shown with the experimental and simulated data, Red Panda makes it possible to perform variant detection in scRNA-seq with higher accuracy as compared to currently available software. Red Panda gains an advantage against other tools by intentionally separating variants into three separate classes and processing them differently: homozygous-looking, bimodally-distributed heterozygous, and non-bimodally-distributed heterozygous.

Using the exome variant data from bulk sequencing as a reference, Red Panda outperforms the other software. It provides both the highest PPV (45\% - Table 1) of any of the tools as well as the highest number of variants in concordance with the exome (913 on average - Fig. 3a). However, PPV is still low compared to using bulk sequencing data $[34,38]$.

Table 2 Average variant count and standard deviation for each tool

\begin{tabular}{|c|c|c|c|c|c|c|}
\hline & FreeBayes & GATK-HC & GATK-UG & Monovar & Platypus & Red Panda \\
\hline Average & 865.8 & 611.1 & 574.7 & 3515.44 & 315.4 & 1071.8 \\
\hline Stdev & 235.3 & 195.1 & 170.5 & 834.04 & 107.1 & 372.6 \\
\hline
\end{tabular}

For this analysis, the total number of variants identified by each tool is reported 


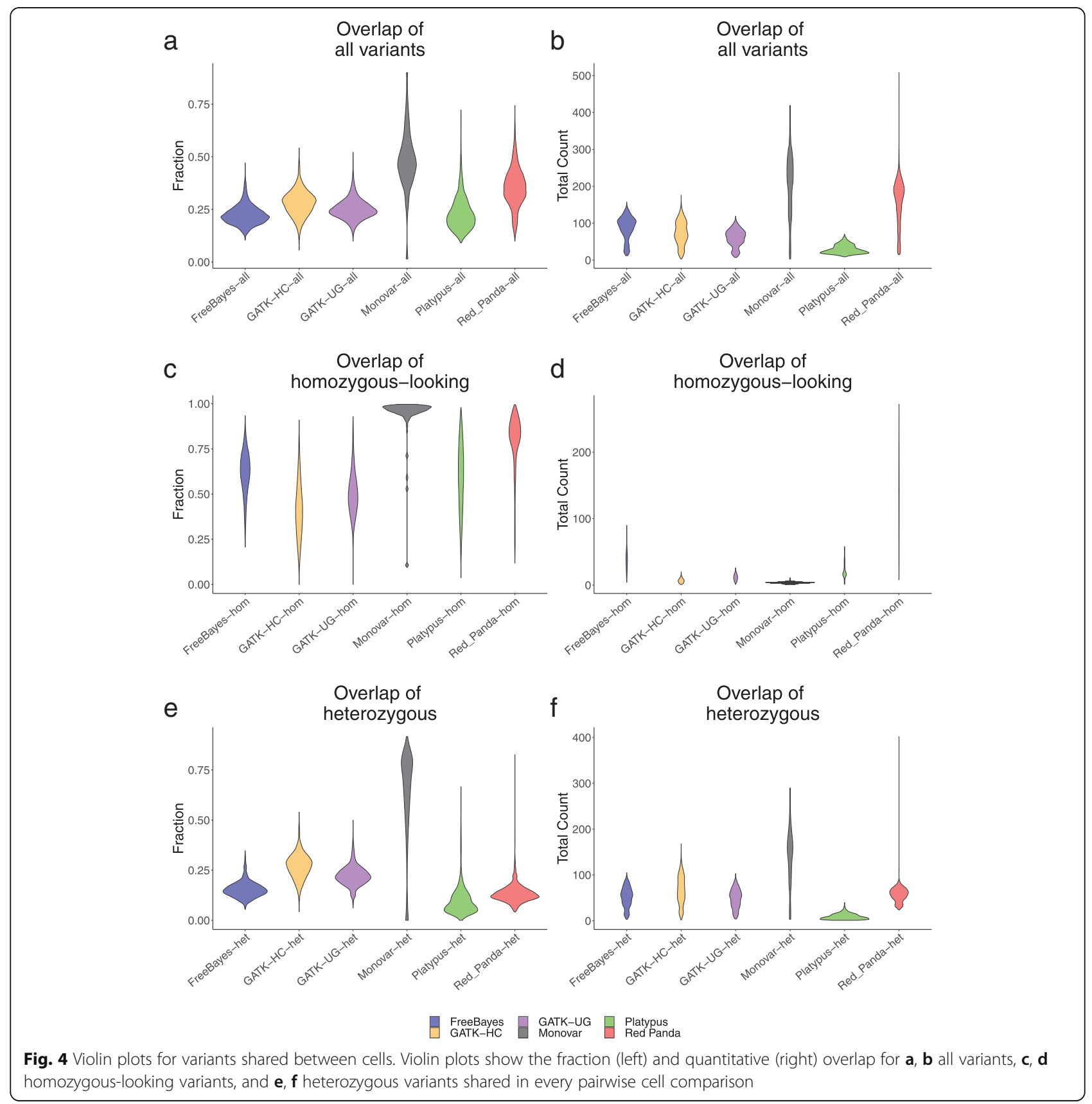

In the MEF sequencing analysis, Red Panda performs better than the four bulk variant callers by identifying the highest number of variants per cell, but does fall short of the total identified by Monovar (Table 2). This could be explained by the fact that Red Panda is designed to work solely on data from individual cells, while Monovar gains an advantage by using variant data from all cells in the sample to calculate a posterior probability for making more confident variant calls. Surprisingly, both Monovar (3515) and FreeBayes (865) identified a much higher number of variants (Table 2) as compared to the results from the human articular chondrocyte data (Fig. 3a). The
FreeBayes results are especially unexpected where it had the fewest number of variants shared between the scRNAseq results and the exome (Fig. 3a). One explanation may be that while FreeBayes identifies a high number of variants, the majority of those are False Positives. This idea is supported by the PPV for FreeBayes from the articular chondrocyte data (Fig. 3c).

The pairwise cellular comparisons (Fig. 4) assessed whether each variant caller performed well based on the consistency of their calls or if they performed poorly, randomly identifying variants in each cell, because, presumably, the same variants should exist in all 55 


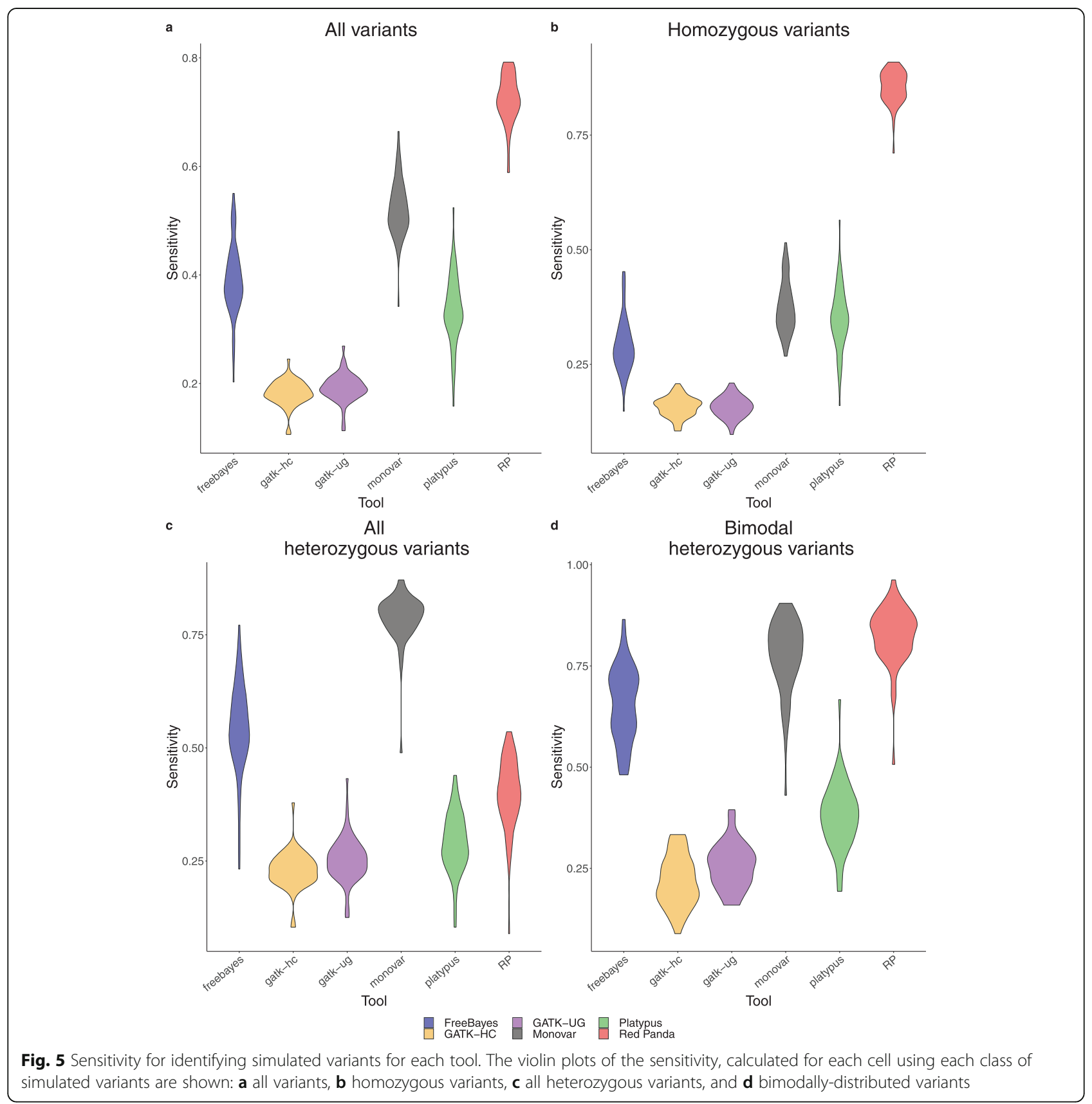

Table 3 Breakdown by tool of variants present in more than one cell

\begin{tabular}{lllllll}
\hline Present in: & FreeBayes & GATK-HC & GATK-UG & Platypus & Red Panda & Intersection of all tools \\
\hline$>=2 / 55$ cells & 2922 & 2463 & 1991 & 2947 & $3159+$ & $96^{*}$ \\
$>=5 / 55$ cells & 970 & 894 & 693 & 324 & 1051 & 18 \\
$>=10 / 55$ cells & 416 & 398 & 309 & 161 & 565 & 0 \\
$>=23 / 55$ of cells & 129 & 122 & 84 & 66 & 257 & 0 \\
$>=42 / 55$ of cells & 38 & 24 & 27 & 22 & 98 & 0 \\
\hline
\end{tabular}

The number of cells in which a variant was found was broken down into five groups: presence in at least 2, 5, 10, 23, or 42 of cells. Additionally, the variants identified by all tools were checked for their presence in the five groups listed above. The variants submitted for Sanger sequencing were drawn from the two groups labeled with a cross ( + ) and an asterisk (*) 
datasets. Red Panda performs extremely well for homozygous-looking variants, but is average for heterozygous variants when compared to the other tools, especially Monovar. This is due to the fact that, while Red Panda in principle confers an algorithmic advantage to identifying heterozygous variants, the monoallelic nature of gene expression and uneven sequencing coverage depth may preclude the tool from realizing its full potential. The majority of the heterozygous variants identified are actually evaluated by GATK HaplotypeCaller, since most are unsupported by a bimodal distribution (Fig. 3b).

The results from the raw number and the fraction of variants overlapping in these pairwise comparisons show that Red Panda has higher distributions in both categories. It is possible to have a large number of variants shared, but also have a smaller fraction of variants shared between cells as is the case with FreeBayes. This indicates that there are more potential False Positives in the data generated by FreeBayes which fits with what was seen in the articular chondrocyte data (Fig. 3c).

The high fraction of homozygous-looking variants shared (Fig. 4c) makes sense as it is less likely that allelic dropout will occur in this class as a result of allelespecific expression making for a more stable population of variants in the scRNA-seq data. Additionally, it is likely that it is rare for any tool to have more than 100 heterozygous variants shared between two cells because of the stochastic nature of allele-specific expression.

It is clearly possible to detect significant variation in transcripts at the single-cell level, but validating those variants at the DNA level is a big challenge due to the inability to isolate sufficient quantities of DNA from the same single cell to perform Sanger sequencing. Hence, we relied on Sanger sequencing performed on a bulk of DNA originating from the population of cells from which we harvested the single cells, which validated only three out of 33 variants tested. It is possible that the variants that failed validation are: False Positives, private mutations to a very small subset of cells that couldn't be captured in the bulk DNA used from the population of cells, or they could be errors introduced in the scRNA-seq library preparation process. Due to these limitations, we relied on the simulated data for MEF cells and paired genomic sequencing as done with the human articular chondrocytes.

There are a number of methods available to imitate read counts and expression profiles [39-41] in scRNAseq; however, there currently exist no tools to generate scRNA-seq reads in silico, making the type of simulation carried out in this study necessary. We created a more realistic environment because the artifacts and flaws inherent to scRNA-seq are considered and maintained by our code. One downside however, is that the dataset used disallows calculating any accuracy statistics requiring False Positive numbers such as specificity and PPV, as the scRNA-seq also contains real variation inherent to the MEFs that will be picked up by each variant caller.

Based on the results from the simulated data (Fig. 5), we found that Red Panda proves its advantage in identifying bimodally-distributed variants as well as homozygous variants, a class of variant that saw other tools struggle. When assessing total heterozygous variants, Monovar is superior to the other tools. This is somewhat surprising as Monovar gains a large part of its advantage in pooling cells together to identify variants, a strategy that should afford it no advantage given our method of simulating variants (each cell was assigned 1000 variants unique to that cell). Another unexpected result was how well FreeBayes performed given what was seen in the results from the human articular chondrocyte experiment where FreeBayes identified very few variants in concordance with the list obtained from exome sequencing, but a clue as to why this is might be found in the results from GATK-HC and GATK-UG. Both of these perform similarly to each other in the simulation with the latter consistently performing slightly better than the former. When variants were called in the exome to generate a reference list against which these variants from the scRNA-seq data could be compared, variants were only retained if they were identified by at least two of the following three tools: FreeBayes, GATK-HC, and Platypus. However, if the variants in this reference list were consistently only supported by the latter two, then it follows that variants identified by FreeBayes in the scRNA-seq experiment would be filtered out and make it appear as though FreeBayes identified a low number of True Positives. This is further supported by the fact that Platypus identifies many more variants in concordance with the exome than FreeBayes. The simulated data indicate that FreeBayes has good sensitivity, but identifies a large set of variants different from both GATK-HC and Platypus. Given the above, in order to improve the accuracy of Red Panda, Monovar or a combination of Monovar and GATK-HC could be used when evaluating nonbimodally-distributed heterozygous variants.

It is important to note that the advantages conferred by Red Panda are currently limited to scRNA-seq generated by library-preparation methods that generate full-length transcripts from cDNA such as Smart-seq2 or Holo-seq [42], although the latter has not been tested in this study.

As methods such as G\&T-seq [43] mature-G\&T-seq produces genomic and transcriptomic sequencing from the same cell-Red Panda can be further validated using sequencing from the same cell, as opposed to scRNA-seq from one cell being compared to exome sequencing from a bulk of cells as was performed with the articular chondrocytes. This would also address the shortcomings tied with validation via Sanger sequencing which uses material from a bulk of cells. 


\section{Conclusions}

Based on the experimental and simulated data, Red Panda provides a distinct advantage over other available software. This improvement comes from its ability to more accurately predict homozygous-looking and bimodally-distributed heterozygous variants as compared to other tools. Due to the unique nature of scRNA-seq data, one must treat heterozygous variants with special consideration, and Red Panda provides a custom approach to this class of variants. From these results, it is clear that due to the inherent nature of RNA expression patterns in single cells, it is difficult to assess what variants exist with the same accuracy that we can with standard exome or WGS. Despite this, Red Panda provides a novel method of identifying variants in scRNAseq and performs this function better than variant callers designed for bulk NGS datasets.

\section{Supplementary Information}

The online version contains supplementary material available at https://doi. org/10.1186/s12864-020-07224-3.

Additional file 1: Supplemental Figure 1. Human articula chondrocyte sequencing strategy. Exome sequencing was paired with scRNA-seq for the the primary tissue culture of human articular chondrocytes. The library prep for the single cells was performed using the updated Smart-seq2 protocol. Supplemental Figure 2. The genomic origin of reads found in each cell. Here one can see what percentage of reads originate from exons (blue), introns (black) or intergenic space (green). The cells A3-C1NC, C10-C64, D12-C72, and H7-C46 have significantly more reads originating outside the exonic region than other samples. Supplemental Figure 3. Expression correlation between articular chondrocytes. Pearson Correlation Coefficient calculated for every possible comparison of cells to each other and the two batches of cells. The darker the color red, the higher the correlation between each cell. "pool26" contains reads pooled from 26 cells, A3-C1NC, C10-C64, D12C72, and H7-C46 were removed; "pool30" contains reads pooled from all 30 cells. as well as identify three other cells that do not correlate well based on their expression patterns: H4-C93NC, G2-C38NC, and E4-C75. Supplemental Figure 4. The sequencing strategy for the MEFs. The MEFs have variant calling performed on them with five variant callers as with the articular chondrocytes. Validation is performed by Sanger sequencing on 40 variants as well as using simulations based on this sequencing data. Supplemental Figure 5. The genomic origin of reads found in each MEF. Here one can see what percentage of reads originate from exons (blue), introns (black) or intergenic space (green). The cell C47 is the only cell to have significantly more reads originating outside the exonic region than other samples. Supplemental Figure 6. Expression correlation between normal MEFs. Pearson Correlation Coefficient calculated for every possible comparison of cells to each other for the normal MEFs. The darker the color red, the higher the correlation between each cell. One can clearly see one cell that fails to correlate will with the other cells: C07. The bottom block of cells significantly correlates with a high number of cells and they are therefore retained. Supplemental Figure 7 Proof of concept data in articular chondrocytes. An example of the variations, from gene CWC22, that we find in the scRNA-seq data as compared to the exome. The main area of interest is the coverage track (the gray histograms). Red corresponds to $T$ and blue corresponds to a $C$. When there are two colors, the top color corresponds to the alternate allele. (a) Two hetSNVs found in the cell A7-C6 have reads supporting them at percentages of $80 \%$ (left) and $20 \%$ (right). The same hetSNVs are found in the exome data at $50 \%$. There is also a homozygous variant (middle) seen in both. (b) One hetSNV found in the same gene at 53\% in the cell A7-C6 is absent in the exome sequencing. This is expected as it does not fit the existing biomodal distribution at $80 \%$ or $20 \%$. Supplemental Figure 8. Workflow for inserting simulated variants. To assess each tool, 1000 simulated variants (650 homozygous, 280 heterozygous, and $\sim 70$ bimodally-distributed heterozygous) were inserted into the alignments for each cell. Standard variant calling was then performed using each tool, and these results were compared to the list of known variants to assess their performance. Supplemental Figure 9. UpSet plots of the overlap between each tool. The overlap of the variants identified by each tool can be seen for the cell G1-C37. Each column of the X-axis shows the overlap between each tool represented by a filled-in dot. For example, the first column indicates that GATK-HC, Monovar, and Red Panda shared 540 variants, the second shows that Red Panda and Monovar share 208 variants, the third column indicates that there were 118 variants shared between Platypus, GATK-HC, Monovar, and Red Panda, and so on. Supplemental Figure 10. The fraction of overlap in variants for every cell using FreeBayes, GATK HC, and GATK UG. The fraction of overlap for (a-c) FreeBayes, (d-f) GATK-HaplotypeCaller, and (g-i) GATKUnifiedGenotyper when comparing $(a, d, g)$ all variants, $(b, e, h)$ homozygouslooking variants, and $(c, f, i)$ heterozygous variants. Each box in the matrix is a comparison between two cells. Supplemental Figure 11. The fraction of overlap in variants for every cell using Monovar, Platypus, and Red Panda. The fraction of overlap for (a-c) Monovar, (d-f) Platypus, and ( $g-i)$ Red Panda when comparing $(a, d, g)$ all variants, $(b, e, h)$ homozygouslooking variants, and $(c, f, i)$ heterozygous variants. Each box in the matrix is a comparison between two cells. Supplemental Figure 12. Raw counts of True Positives for each tool. The box plots of the raw number of True Positives show how well each tool is at identifying variants in the simulation. Due to advantages gained in identifying homozygous and bimodally-distributed variants, Red Panda identifies the highest number of True Positives. Supplemental Table 1. Eight human articular chondrocytes removed for quality reasons. "Too many reads outside exon" is defined as one standard deviation above the median percentage of reads aligned outside exons for all samples. Statistically insignificant correlation coefficient is defined as $p>0.05$ for a Pearson correlation coefficient when comparing the transcription profile of a single cell to the pool of all 30 cells. Supplemental Table 2. Parameters used to design the primers used for PCR and Sanger. Parameters with a *were changed from their defaults to ensure good sequencing. Supplemental Table 3. Human articular chondrocyte exome sequencing statistics. Sequencing and analysis statistics of the exome data from the human articular chondrocytes. Supplemental Table 4. Human articular chondrocyte exome variant calling statistics. Variant analysis statistics of the exome data from the human articular chondrocytes using the ensemble approach where 2/3 variant caller tools had to agree to call a variant. Supplemental Table 5. Summary of the human articular chondrocytes captured on the C1. Supplemental Table 6. Summary table of variants identified by Red Panda in human articular chondrocytes. Percent of variants that are homozygous-looking, heterozygous, heterozygous and validated by GATK-HC, heterozygous and validated by Red Panda are calculated. Average total number of variants in the final VCF file is also shown. Supplemental Table 7. Validation of variants identified by all five variant callers. In the Validated by Sanger column, N = No, and NS = No Sequence at that position. Supplemental Table 8. First sequencing pass: Validation of variants only identified by Red Panda. In the Validated by Sanger column, $Y=Y e s, N=$ No, and NS = No Sequence at that position. Supplemental Table 9. Second sequencing pass: Validation of variants only identified by Red Panda. In the Validated by Sanger column, $Y=Y e s, N=$ No, and NS = No Sequence at that position

\section{Abbreviations}

scRNA-seq: Single-cell RNA sequencing; SCS: Single-cell sequencing; MEFs: Mouse embryonic fibroblasts; SNVs: Single nucleotide variants; PPV: Positive predictive value; WGS: Whole-genome sequencing

\section{Acknowledgements}

The authors are very grateful to Kristin Wipfler for her valuable input during algorithm development, Cecily Zdan for copy editing, and Dr. James Eudy for his valuable input during setup and processing of the sequencing. 


\section{About this supplement}

This article has been published as part of BMC Genomics Volume 21 Supplement 11 2020: Bioinformatics methods for biomedical data science. The full contents of the supplement are available at https://bmcgenomics. biomedcentral.com/articles/supplements/volume-21-supplement-11.

\section{Authors' contributions}

AC designed the study, performed all analyses, and wrote the manuscript. $\mathrm{SR}, \mathrm{KS}$, and SP performed experiments. CG with the help of NKM supervised the project, provided essential feedback on experimental results and input on the analyses themselves, and thoroughly edited the manuscript. KB and $A D$ provided technical guidance and materialistic support for experiments performed by SR/SP and KS, respectively. All authors read and approved the final manuscript.

\section{Funding}

This work was supported by the development funds to CG, and the Office of Graduate Studies fellowship to AC, from the University of Nebraska Medical Center (UNMC) and the NIH award [2P01AG029531] to CG. The University of Nebraska DNA Sequencing Core and the Bioinformatics and Systems Biology core receive partial support from the National Institutes of Health grants [P20GM103427, 1P30GM110768, P30CA036727]. Publication costs are funded by the development funds to CG from UNMC.

\section{Availability of data and materials}

Red Panda is released under the MIT license and can be found on GitHub at https://github.com/adambioi/red_panda. Manual and source code of the tools are available in the GitHub repository.

\section{Ethics approval and consent to participate}

Articular chondrocytes were harvested from a Caucasian female patient undergoing total knee replacement, who provided informed consent under the University of Nebraska Medical Center Institutional Review Board (IRB) \#691-13-EP before the study.

\section{Consent for publication}

Not applicable.

\section{Competing interests}

The authors declare that the research was conducted in the absence of any commercial or financial relationships that could be construed as a potential conflict of interest.

\section{Published: 29 December 2020}

\section{References}

1. Navin N, Kendall J, Troge J, Andrews P, Rodgers L, Mclndoo J, Cook K, Stepansky A, Levy D, Esposito D, et al. Tumour evolution inferred by singlecell sequencing. Nature. 2011;472(7341):90-4.

2. Suzuki A, Matsushima K, Makinoshima H, Sugano S, Kohno T, Tsuchihara K, Suzuki Y. Single-cell analysis of lung adenocarcinoma cell lines reveals diverse expression patterns of individual cells invoked by a molecular target drug treatment. Genome Biol. 2015;16:66.

3. McConnell MJ, Lindberg MR, Brennand KJ, Piper JC, Voet T, Cowing-Zitron C, Shumilina S, Lasken RS, Vermeesch JR, Hall IM, et al. Mosaic copy number variation in human neurons. Science. 2013;342(6158):632-7.

4. Ramsköld D, Luo S, Wang Y-C, Li R, Deng Q, Faridani OR, Daniels GA Khrebtukova I, Loring JF, Laurent LC, et al. Full-length mRNA-Seq from single-cell levels of RNA and individual circulating tumor cells. Nat Biotechnol. 2012;30(8):777-82.

5. Ni X, Zhuo M, Su Z, Duan J, Gao Y, Wang Z, Zong C, Bai H, Chapman AR, Zhao J, et al. Reproducible copy number variation patterns among single circulating tumor cells of lung cancer patients. Proc Natl Acad Sci U S A. 2013:110(52):21083-8

6. Gawad C, Koh W, Quake SR. Dissecting the clonal origins of childhood acute lymphoblastic leukemia by single-cell genomics. Proc Natl Acad Sci U S A. 2014;111(50):17947-52

7. Jan M, Snyder TM, Corces-Zimmerman MR, Vyas P, Weissman IL, Quake SR, Majeti R. Clonal evolution of preleukemic hematopoietic stem cells precedes human acute myeloid leukemia. Sci Transl Med. 2012:4(149): 149 ra118.
8. Lodato MA, Woodworth MB, Lee S, Evrony GD, Mehta BK, Karger A, Lee S, Chittenden TW, D'Gama AM, Cai X, et al. Somatic mutation in single human neurons tracks developmental and transcriptional history. Science. 2015; 350(6256):94-8.

9. Grün D, Lyubimova A, Kester L, Wiebrands K, Basak O, Sasaki N, Clevers H, van Oudenaarden A. Single-cell messenger RNA sequencing reveals rare intestinal cell types. Nature. 2015:525(7568):251-5.

10. Jaitin DA, Kenigsberg E, Keren-Shaul H, Elefant N, Paul F, Zaretsky I, Mildner A, Cohen N, Jung S, Tanay A, et al. Massively parallel single-cell RNA-seq for marker-free decomposition of tissues into cell types. Science. 2014; 343(6172):776-9.

11. Zeisel A, Muñoz-Manchado AB, Codeluppi S, Lönnerberg P, La Manno G, Juréus A, Marques S, Munguba H, He L, Betsholtz C, et al. Brain structure. Cell types in the mouse cortex and hippocampus revealed by single-cell RNA-seq. Science. 2015;347(6226):1138-42.

12. Tennessen JA, Bigham AW, O'Connor TD, Fu W, Kenny EE, Gravel S, McGee S, Do R, Liu X, Jun G, et al. Evolution and functional impact of rare coding variation from deep sequencing of human exomes. Science. 2012;337(6090):64-9.

13. Gill R, Cheung YH, Shen Y, Lanzano P, Mirza NM, Ten S, Maclaren NK, Motaghedi R, Han JC, Yanovski JA, et al. Whole-exome sequencing identifies novel LEPR mutations in individuals with severe early onset obesity. Obesity. 2014:22(2):576-84.

14. Ku C-S, Tan EK, Cooper DN. From the periphery to Centre stage: de novo single nucleotide variants play a key role in human genetic disease. J Med Genet. 2013;50(4):203-11.

15. Lek M, Karczewski KJ, Minikel EV, Samocha KE, Banks E, Fennell T, O'DonnellLuria $\mathrm{AH}$, Ware JS, Hill AJ, Cummings BB, et al. Analysis of protein-coding genetic variation in 60,706 humans. Nature. 2016;536(7616):285-91.

16. Sheng Q, Zhao S, Li Cl, Shyr Y, Guo Y. Practicability of detecting somatic point mutation from RNA high throughput sequencing data. Genomics. 2016:107(5):163-9.

17. Xu C. A review of somatic single nucleotide variant calling algorithms for next generation sequencing data. Comput Struct Biotechnol J. 2018;16:15-24.

18. Zafar H, Wang Y, Nakhleh L, Navin N, Chen K. Monovar: single-nucleotide variant detection in single cells. Nat Methods. 2016;13(6):505-7.

19. Borel C, Ferreira PG, Santoni F, Delaneau O, Fort A, Popadin KY, Garieri M, Falconnet E, Ribaux P, Guipponi M, et al. Biased allelic expression in human primary fibroblast single cells. Am J Hum Genet. 2015;96(1):70-80.

20. Garrison E, Marth G: Haplotype-based variant detection from short-read sequencing. 2012.

21. Poplin R, Ruano-Rubio V, DePristo MA, Fennell TJ, Carneiro MO, Van der Auwera GA, Kling DE, Gauthier LD, Levy-Moonshine A, Roazen D et al: Scaling accurate genetic variant discovery to tens of thousands of samples. 2017.

22. DePristo MA, Banks E, Poplin R, Garimella KV, Maguire JR, Hartl C, Philippakis AA, del Angel G, Rivas MA, Hanna M, et al. A framework for variation discovery and genotyping using next-generation DNA sequencing data. Nat Genet. 2011;43(5):491-8.

23. Rimmer A, Phan H, Mathieson I, labal Z, Twigg SRF, Consortium WGS, Wilkie AOM, MCVean G, Lunter G. Integrating mapping-, assembly- and haplotypebased approaches for calling variants in clinical sequencing applications. Nat Genet. 2014:46(8):912-8.

24. Wang Y, Navin NE. Advances and applications of single-cell sequencing technologies. Mol Cell. 2015;58(4):598-609.

25. Cornish A, Roychoudhury S, Sarma K, Pramanik S, Bhakat K, Dudley A, Mishra NK, Guda C: Red Panda: A novel method for detecting variants in single-cell RNA sequencing. bioRxiv 2020:2020.2001.2008.898874

26. Xu J: Preparation, culture, and immortalization of mouse embryonic fibroblasts. Curr Protoc Mol Biol 2005, Chapter 28:Unit 28.21

27. Patro R, Mount SM, Kingsford C. Sailfish enables alignment-free isoform quantification from RNA-seq reads using lightweight algorithms. Nat Biotechnol. 2014;32(5):462-4

28. Li H. A statistical framework for SNP calling, mutation discovery, association mapping and population genetical parameter estimation from sequencing data. Bioinformatics. 2011:27(21):2987-93.

29. Yan H. Allelic variation in human gene expression. Science. 2002;297(5584): $1143-3$

30. Gregg C, Zhang J, Weissbourd B, Luo S, Schroth GP, Haig D, Dulac C. Highresolution analysis of parent-of-origin allelic expression in the mouse brain. Science. 2010;329(5992):643-8 
31. Marinov GK, Williams BA, McCue K, Schroth GP, Gertz J, Myers RM, Wold BJ From single-cell to cell-pool transcriptomes: stochasticity in gene expression and RNA splicing. Genome Res. 2014;24(3):496-510.

32. Deng $Q$, Ramsköld D, Reinius B, Sandberg R. Single-cell RNA-seq reveals dynamic, random monoallelic gene expression in mammalian cells. Science. 2014;343(6167):193-6.

33. Gimelbrant A, Hutchinson JN, Thompson BR, Chess A. Widespread monoallelic expression on human autosomes. Science. 2007;318(5853): $1136-40$

34. Cornish A, Guda C. A comparison of variant calling pipelines using genome in a bottle as a reference. Biomed Res Int. 2015;2015:1-11.

35. Dewey FE, Murray MF, Overton JD, Habegger L, Leader JB, Fetterolf SN, O'Dushlaine C, Van Hout CV, Staples J, Gonzaga-Jauregui C, et al. Distribution and clinical impact of functional variants in 50,726 wholeexome sequences from the DiscovEHR study. Science. 2016:354(6319): aaf6814.

36. Genomes Project C, Abecasis GR, Altshuler D, Auton A, Brooks LD, Durbin RM, Gibbs RA, Hurles ME, McVean GA. A map of human genome variation from population-scale sequencing. Nature. 2010;467(7319):1061-73.

37. Choi M, Scholl UI, Ji W, Liu T, Tikhonova IR, Zumbo P, Nayir A, Bakkaloğlu A, Özen S, Sanjad S, et al. Genetic diagnosis by whole exome capture and massively parallel DNA sequencing. Proc Natl Acad Sci. 2009;106(45):19096-101.

38. Sandmann S, de Graaf AO, van der Reijden BA, Jansen JH, Dugas M. GLMbased optimization of NGS data analysis: a case study of Roche 454, ion torrent PGM and Illumina NextSeq sequencing data. PLoS One. 2017;12(2): e0171983.

39. Zappia L, Phipson B, Oshlack A. Splatter: simulation of single-cell RNA sequencing data. Genome Biol. 2017;18(1):174.

40. Risso D, Perraudeau F, Gribkova S, Dudoit S, Vert J-P. A general and flexible method for signal extraction from single-cell RNA-seq data. Nat Commun. 2018;9(1):284.

41. Severson DT, Owen RP, White MJ, Lu X, Schuster-Böckler B. BEARscc determines robustness of single-cell clusters using simulated technical replicates. Nat Commun. 2018;9(1):1187.

42. Xiao Z, Cheng G, Jiao Y, Pan C, Li R, Jia D, Zhu J, Wu C, Zheng M, Jia J. Holo-Seq: single-cell sequencing of holo-transcriptome. Genome Biol. 2018; 19(1):163.

43. Macaulay IC, Haerty W, Kumar P, Li YI, Hu TX, Teng MJ, Goolam M, Saurat N, Coupland P, Shirley LM, et al. G\&T-seq: parallel sequencing of single-cell genomes and transcriptomes. Nat Methods. 2015;12(6):519-22.

\section{Publisher's Note}

Springer Nature remains neutral with regard to jurisdictional claims in published maps and institutional affiliations.

Ready to submit your research? Choose BMC and benefit from:
- fast, convenient online submission
- thorough peer review by experienced researchers in your field
- rapid publication on acceptance
- support for research data, including large and complex data types
- gold Open Access which fosters wider collaboration and increased citations
- maximum visibility for your research: over 100M website views per year
At BMC, research is always in progress.
Learn more biomedcentral.com/submissions

